\title{
Conductance of Threading Dislocations in InGaAs/Si stacks by temperature-CAFM measurements
}

\author{
C. Couso, V. Iglesias, M. Porti, S. Claramunt, M. Nafría, N. Domingo, A. Cordes and G. Bersuker.
}

\begin{abstract}
The stacks of III-V materials, grown on the Si substrate, that are considered for fabrication of highly scaled devices tend to develop structural defects, in particular Threading Dislocations (TDs), which affect device electrical properties. We demonstrate that the characteristics of TD sites can be analyzed by utilizing the Conductive Atomic Force Microscopy (CAFM) technique with nanoscale spatial resolution within a wide temperature range. In the studied InGaAs/Si stacks, electrical conductance through TD sites was found to be governed by the Poole-Frenkel emission, while off-TDs conductivity is dominated by the Thermionic Emission process.
\end{abstract}

Index Terms - CAFM, semiconductor defects, threading dislocation, thermionic emission, poole frenkel emission.

\section{INTRODUCTION}

High mobility channel transistors for complex multi-device circuitries are considered to be fabricated using III-V materials locally grown over the $\mathrm{Si}$ substrate. Due to the lattice mismatch between these materials, the III-V films tend to form structural defects [1], in particular Threading Dislocations (TDs), which may contribute to charge carriers transport thus degrading device performance [2], [3]. In particular, TDs enhance current conduction in forward and reverse biased diodes [4] and increase device-to-device variability due to the formation of multiple parallel paths with different conductivity (on- and off-TD sites) [5]. In HEMTs, TDs increase reverse gate leakage current and frequency related noise (affecting, for example, low-noise amplifiers [3]) and reduce drain saturation current, peak transconductance, off-state breakdown voltage and cutoff frequency [3]. In InGaAs nMOSFETs, TDs severely reduce carrier mobility [6], [7], affecting $\mathrm{I}_{\mathrm{ON}}$ and $\mathrm{V}_{\mathrm{TH}}$ and limiting drive current. Therefore, monitoring the TDs' contribution/effect to the charge transport through the III-V channels is critical for controlling/improving device characteristics [3], [7].

Electrical conduction through III-V films has been previously studied on fully processed devices by measuring I$\mathrm{V}$ characteristics [1], [8], [9]. However, these device level measurements register the overall current through the entire device area that may mask the current component associated

Manuscript received October 9, 2001. (Write the date on which you submitted your paper for review.) This work has been partially supported by the Spanish MINECO and ERDF (TEC2013-45638-C3-1-R), the Generalitat de Catalunya (2014SGR-384).

C. Couso, V. Iglesias, M. Porti, S. Claramunt and M. Nafría are with Dept. Enginyeria Electrònica, Campus UAB, 08193 Bellaterra (Spain). (e-mail: carlos.couso@uab.cat).

N. Domingo is with the Institut Català de Nanociència i Nanotecnologia (ICN2), Campus UAB, 08193 Bellaterra (Spain).

A. Cordes is with the Sematech, Austin, TX, (USA).

G. Bersuker is with the The Aerospace Corporation, Los Angeles, (USA) with TDs. Therefore, since TDs cross-section dimensions can be in the nanometer range, employing techniques with the nanoscale spatial resolution capability, such as the Conductive Atomic Force Microscopy (CAFM), is expected to provide TD-specific conductivity data. Indeed, the CAFM technique has been widely used for nanoscale electrical characterization of the defects in gate dielectrics, either grown or generated under the applied electrical stress and irradiation [10]-[17], as well as TDs in III-V based stacks. In the latter, the I-V measurements were performed on the TD sites attributed to the surface pits (as was observed in the CAFM topographical images). These sites exhibited lower turn-on voltages and higher leakage currents under the forward and reverse biases [4], [5], [18]-[20]. Higher forward currents through TDs was attributed to Poole-Frenkel (PF) conduction [19], [20] and described as caused by lowered barrier heights of the Schottky contacts between the tip and samples [5].

In the above studies, CAFM measurements were performed at ambient temperatures, and, in some instances, were combined with the device level I-V characteristics collected at different temperatures, which allowed attributing the reverse current to the PF emission [9], [21]. However, the device-level $\mathrm{I}-\mathrm{V}$ data could not be linked exclusively to the TDs electrical properties because they were also affected by contributions from the electrically active non-TD device area. The present study extends the analysis by performing CAFM measurements of the localized (contact site specific) currentvoltage characteristics, on both on-TD and off-TD sites in the III-V/Si stack, at different temperatures.

\section{SAMPLE PREPARATION AND CHARACTERIZATION}

The CAFM measurements were performed with an Asylum MFP3D System, using ORCA module with a Polyheater sample stage. This configuration allows for conductivity measurements (from $100 \mathrm{pA}$ to $10 \mu \mathrm{A}$ ) while keeping the tip grounded and in a temperature range up to $300^{\circ} \mathrm{C}$. The sample investigated with CAFM consists of a Te:InGaAs 30 nm/InGaAs 120 nm/InP 600 nm/GaAs 500 nm/Si stack. Fig. 1 (a) shows a cross section TEM image of a studied stack. In Fig. 1 (a), structural defects (TDs) can be observed propagating from the interface with a $\mathrm{Si}$ substrate and may extend significantly through the III-V material thickness, in some instances reaching the film surface.

To identify the TDs locations on the surface, topographical and current maps were measured simultaneously by scanning the CAFM tip on randomly selected surface areas of the stack. Fig. 1 shows (b) topographical and (c) current maps at $2 \mathrm{~V}$, injection from the tip. Detected surface pits in Fig. 1 (b) 
(attributed to TDs) match those of the high conduction sites in the current map. I-V curves on the targeted defect sites were then collected using ramped voltage measurements. The tipsample contact was characterized as being of the Schottky type [22], although currents under the reverse bias condition partially cover the rectifying features. (a)

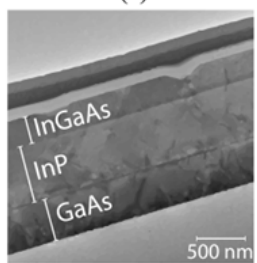

(b)

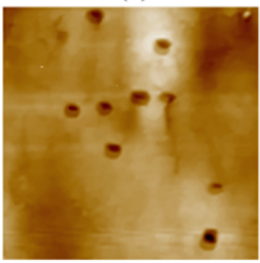

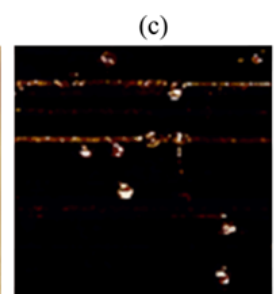

$10 \mathrm{nA}$
Fig. 1. (a) TEM cross section image. (b) Topographical and (c) reverse current (measured at $2 \mathrm{~V}$ ) CAFM images collected on $7.5 \times 7.5 \mu \mathrm{m}^{2}$ area. Two bright lines in the upper region of a current map (c) correspond to AFM artifacts.

To compare temperature dependencies of the conduction through on-TDs and off-TDs sites, CAFM I-V measurements were performed at several temperatures $(313,348,398$ and $448 \mathrm{~K})$ at both sites. It is important to note that the CAFMbased I-V measurements at different temperatures are technically rather complex to execute. To change temperature, the tip must be withdrawn from the sample surface, and then it has to be repositioned on the same site, which is a challenging task in the case of the required highly scaled area range. On the other hand, the measurement itself could modify the TD electrical properties: changes in electrical conductivity, observed after the site was re-measured at different temperatures, might be dominated by structural changes (charge trapping) induced by previous measurements on this site [23]. To mitigate such uncertainty, a statistical analysis of the electrical conductivity data collected at each temperature on different TD sites was carried out (I-V curves were not measured repeatedly on the same site). This method was found to be more accurate than measuring a fixed TD site at different temperatures. In order to ensure the stability of tip properties during the experiments, the Pt bulk tips were used.

\section{RESULTS AND DISCUSSION}

Fig. 2 (a) shows examples of I-V curves measured over the TDs (on-TDs sites) at 4 different temperatures within the 313 K-448 K range. Similar measurements have been performed over the regular (off-TDs) surface sites. Comparisons of the I$\mathrm{V}$ curves measured on-TDs (open symbols) and off-TDs (solid symbols) at 313 and $448 \mathrm{~K}$, Fig. 2(b), demonstrates that the onTD currents are higher than those of the off-TDs, consistent with the data in the images in Fig. 1. Moreover, currents through the sites and, subsequently, their conductivities, increase with temperature, more pronouncedly in the on-TD sites. Assuming that higher current values over the on-TD sites (compared to the off-TDs) are associated with their structural defects, the measured I-V dependencies were modeled using the Poole-Frenkel (PF) emission process [19]:

$$
J=C \times E \times \exp \left\{-q / K T\left(\phi_{t}-\sqrt{q E_{b} / \pi \varepsilon_{0} \varepsilon_{s}}\right)\right\}
$$

where $J$ is the current density, $E$ the electric field over the energy barrier at the tip-semiconductor interface, $q$ is the electron charge, $K$ is the Boltzmann constant, $\varepsilon_{0}$ and $\varepsilon_{s}$ are the vacuum and relative dielectric permittivity (at high frequency) of the semiconductor, respectively, $T$ is the temperature, $\Phi_{t}$ is the barrier height for electron emission from the trapped state and $C$ is a constant. The electric field dependency in the PF process can be linearized when plotting (1) as:

$$
\begin{gathered}
\ln (J / E)=R(T) \times \sqrt{E}+S(T) \\
R(T)=q / K T\left(\sqrt{q E / \pi \varepsilon_{0} \varepsilon_{s}}\right) \\
S(T)=-q \phi_{t} / K T+\ln (C)
\end{gathered}
$$

where $R(T)$ and $S(T)$ are the slope and y-intercept, respectively.
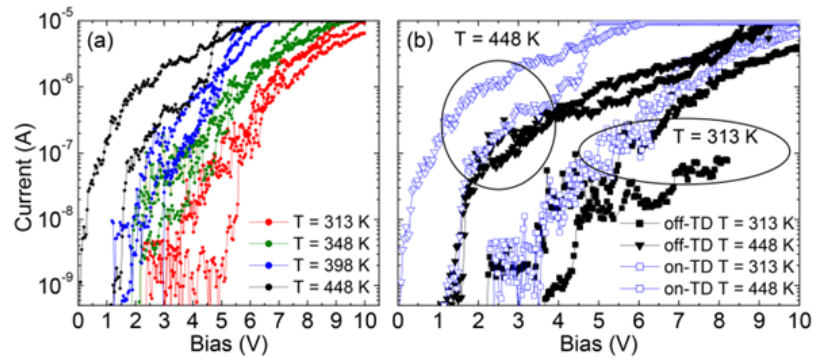

Fig. 2. (a) A set of $I-V$ curves measured on different TDs sites (same temperature color). (b) Example of I-V curves measured on-TDs (open symbols) and off-TDs (solid symbols) at $313 \mathrm{~K}$ and $448 \mathrm{~K}$.

The I-V curves in Fig. 2 (a) are re-plotted following the expressions of (2-4) (see Fig. 3 (a), a single I-V curve is shown for each temperature). At each temperature, the measured (re-plotted) I-V dependencies generally follow (although they fluctuate) a trend of (2) (shown by the linear lines in Fig. 3 (a) suggesting that the conduction through the TDs is consistent with the PF mechanism (1).
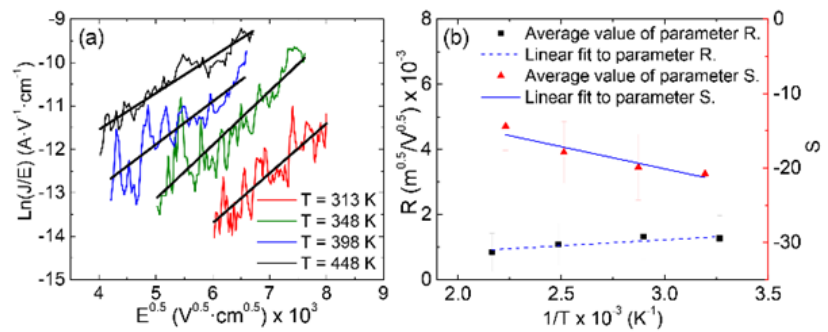

Fig. 3. (a) Measured I-V curves in Fig. 2 (a) are plotted (one curve per each T) following the expressions (2-4). The linear lines represent the fittings using (24). (b) R(T) (squares) and S(T) (triangles) mean values in the y-axis, which are the slope and the y-intercept values of the entire set of measured I-V curves respectively, as a function $1 / \mathrm{T}$. Continuous lines show the linear dependency of $\mathrm{R}(\mathrm{T})$ and $\mathrm{S}(\mathrm{T})$ vs. $1 / \mathrm{T}$ in (3 and 4$)$.

The average values of $S(T)$, the $y$-intercept values of the fit (solid) lines in Fig. 3 (a), are plotted as a function of $1 / \mathrm{T}$ (triangles) in Fig. 3 (b). The $\mathrm{S}(\mathrm{T})$ linear dependency on $1 / \mathrm{T}$ is reflected by a solid line. The $\mathrm{S}(\mathrm{T})$ slope is proportional to the emission barrier height $\Phi_{t}(4)$, which calculated value of 0.51 $\mathrm{eV}$ is compatible to the reported ones [24]. Similarly, the slopes of the fit lines in Fig. 3 (a), R(T), also show a linear dependency on $1 / \mathrm{T}$ (squares) in Fig. 3 (b). Using (3), $\varepsilon_{\mathrm{s}}$ is 
estimated to be about 4.52. It must be noted that the calculated values of $\Phi_{\mathrm{t}}$ and $\varepsilon_{\mathrm{s}}$ have been obtained from a linear fitting of the $S(T)$ and $R(T)$ average values at different temperatures. The $\mathrm{S}(\mathrm{T})$ and $\mathrm{R}(\mathrm{T})$ average values (at a given $\mathrm{T}$ ) used in the linear fitting correspond to the average values of $\mathrm{S}$ and $\mathrm{R}$ obtained from the data collected on different TDs sites. Therefore, the calculated values of $\Phi_{\mathrm{t}}$ and $\varepsilon_{\mathrm{s}}$ correspond to an average value of the analyzed sites.

To verify the validity of the PF-based mechanism in TDs, the current in (1) was calculated using the average value of the physical parameters in (3 and 4). Fig. 4 (a) shows the experimental $\mathrm{I}-\mathrm{V}$ curves measured at different temperatures (symbols) and I-V dependencies calculated using the PF equation (solid lines) with the experimentally determined parameters, which includes only one I-V curve for each $\mathrm{T}$ of Fig. 2. The data at low biases (noise level of the setup) and very high biases (possible parasitic), is not shown. I-V trends based on the PF emission mechanism fit well the measured ones.

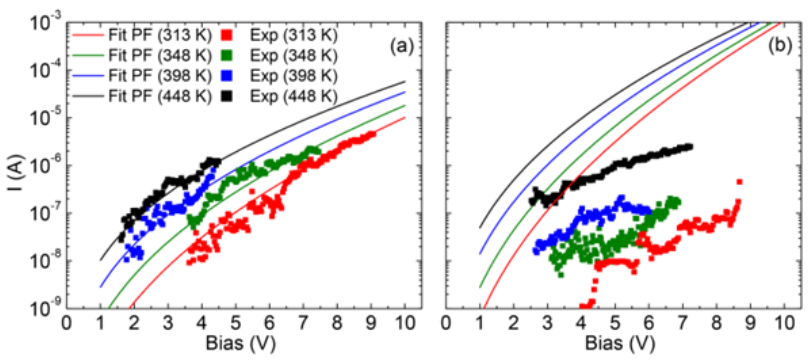

Fig. 4. I-V curves (symbols) measured on-TD (a) and off-TD (b) sites at different temperatures. Solid lines correspond to the calculated PF current using the parameters obtained by the fitting of $S(T)$ and $R(T)$.

Although the on-TDs conduction is generally higher than that of the background areas, the off-TD conductivity is not negligible (see Fig. 2 (b)) and, therefore, needs to be evaluated. The above presented procedure was applied to the data in Fig. $2(\mathrm{~b})$, yielding $\Phi_{\mathrm{t}}=0.53 \mathrm{eV}$ and $\varepsilon_{\mathrm{s}}=2.56$ values. However, the I-V curves calculated by using these parameters do not match the experimental data (see Fig. 4 (b)). Hence, the above analysis demonstrates that the PF emission is dominant process exclusively in the TD sites.

$\mathrm{I}-\mathrm{V}$ curves measured under the reverse-bias on the off-TD sites were fitted by the Thermionic / Schottky Emission (TE) mechanism:

$$
J=A^{*} \times T^{2} \times \exp \left\{-q / K T\left(\phi_{b}-\sqrt{q E / 4 \pi \varepsilon_{0} \varepsilon_{s}}\right)\right\}
$$

Here $A^{*}$ is the effective Richardson constant, $\Phi b$ is the Schottky barrier height and $\mathrm{E}$ the electric field over the energy barrier at the tip-semiconductor interface (related to the bias applied to the structure); remaining parameters are defined previously. Thus, using a procedure similar to that used in the on-TDs case can be followed to estimate $\Phi b$ in $S^{\prime}(T)$. Since TE process is effective predominantly at high temperatures, only the data measured at 348, 398 and $448 \mathrm{~K}$ were considered. The barrier height and permitivity for the off-TD sites were found to be $\Phi b=0.61 \mathrm{eV}$ and $\varepsilon_{\mathrm{s}}=3.9$, respectively.

Fig. 5 (b) shows experimental off-TD I-V curves (symbols) and calculated TE current for each temperature (solid lines) using (5) and the values obtained from the experimental data $\left(\Phi_{\mathrm{b}}=0.61 \mathrm{eV}\right.$ and $\left.\varepsilon_{\mathrm{s}}=3.9\right)$. Note that the experimental data matches very well to the TE-based calculations (especially at a higher temperature, when the TE process dominates). Therefore, off-TD current could be described by considering electrons tunneling over the barrier when the process of the defects assisted electron transport can be negligible. The observation of high off-TDs currents under the reverse bias conditions could be understood by considering a low barrier height for the electrons injected from the metal $\left(\Phi_{\mathrm{b}}=0.61\right.$ $\mathrm{eV}$ ), which depends, in our case, on the materials in a CAFM tip and top layer of the analyzed sample.
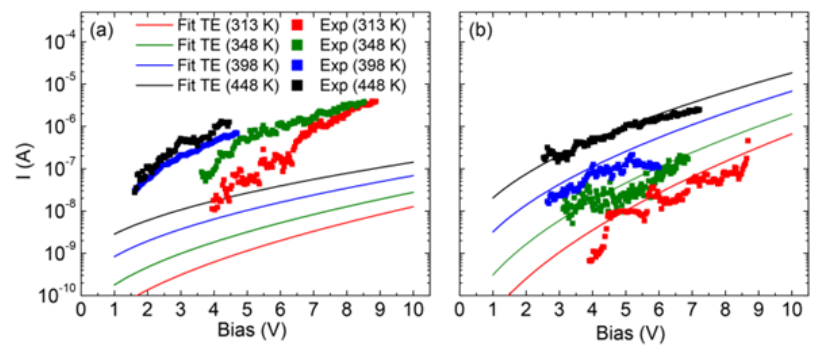

Fig. 5. I-V curves (symbols) measured on the on-TDs (a) and off-TD (b) sites at different temperatures. Solid lines correspond to the calculated TE current using the parameters obtained by the fittings of S'(T) and R'(T).

Following the procedure employed above in the case of the on-TDs I-V curves for the TE emission, $\Phi_{\mathrm{b}}=0.37 \mathrm{eV}$ and $\varepsilon_{\mathrm{s}}=$ 11.7 were obtained, respectively. However, the I-V curves calculated by using these parameters in (5) do not match the experimental data (Fig. 5 (a)). This result further supports the conclusion that the conduction through the on-TD sites can be described by considering the PF emission, but not the TE process, pointing-out that TDs are a major component of enhanced reverse-leakage current through III-V materials. Presented data demonstrate that, thanks to the nanoscale resolution, the employed methodology provides a valuable insight to the TDs conduction properties, critical for optimizing multi-level structures of scaled devices.

\section{CONCLUSION}

Conduction through TDs sites in the III-V materials have been investigated using CAFM-based measurements at different temperatures. The conductivity of TDs, observed as the surface pits in topographical maps, is found to be higher than that of the off-TD sites. In both on- and off-TD sites, the currents were observed to increase with temperature. The onTD electrical conduction can be described by accounting for the Poole-Frenkel (PF) emission process suggesting that it can be the dominant conduction mechanism at these sites. Conductivity of the off-TD sites, in particular, at higher temperatures, seems to be controlled by the thermionic emission mechanism. In summary, the results demonstrate that the CAFM technique is well-suited for evaluating the TDs electrical properties with the nanoscale resolution, required to identify material structural features affecting device performance. 


\section{REFERENCES}

[1] J. Yang, S. Cui, T. P. Ma, T.-H. Hung, D. Nath, S. Krishnamoorthy, and S. Rajan, "A study of electrically active traps in AlGaN/GaN high electron mobility transistor," Appl. Phys. Lett., vol. 103, no. 2013, p. 173520, 2013, DOI:10.1063/1.4826922.

[2] Y. Zhang, M. Sun, H. Wong, Y. Lin, P. Srivastava, C. Hatem, M. Azize, D. Piedra, L. Yu, T. Sumitomo, N. A. de Braga, R. V. Mickevicius, and T. Palacios, "Origin and Control of OFF-State Leakage Current in GaN-on-Si Vertical Diodes," IEEE Trans. Electron Devices, vol. 62, no. 7, pp. 2155-2161, 2015, DOI:10.1109/TED.2015.2426711.

[3] Z. H. Feng, S. J. Cai, K. J. Chen, and K. M. Lau, "Enhancedperformance of AlGaN-GaN HEMTs grown on grooved sapphire substrates," IEEE Electron Device Lett., vol. 26, no. 12, pp. 870 872, Dec. 2005, DOI:10.1109/LED.2005.859675.

[4] A. Hofer, G. Benstetter, R. Biberger, C. Leirer, and G. Brüderl, "Analysis of crystal defects on GaN-based semiconductors with advanced scanning probe microscope techniques," Thin Solid Films, vol. 544, pp. 139-143, 2013, DOI:10.1016/j.tsf.2013.04.049.

[5] F. Giannazzo, F. Roccaforte, F. Iucolano, V. Raineri, F. Ruffino, and M. G. Grimaldi, "Nanoscale current transport through Schottky contacts on wide bandgap semiconductors," J. Vac. Sci. Technol. B Microelectron. Nanom. Struct., vol. 27, no. 2009, p. 789, 2009, DOI:10.1116/1.3043453.

[6] P. Goley and M. Hudait, "Germanium Based Field-Effect Transistors: Challenges and Opportunities,” Materials (Basel)., vol. 7, no. 3, pp. 2301-2339, 2014, DOI:10.3390/ma7032301

[7] A. Nayfeh, C. O. Chui, T. Yonehara, and K. C. Saraswat, "Fabrication of high-quality p-MOSFET in Ge Grown heteroepitaxially on Si," IEEE Electron Device Lett., vol. 26, no. 5, pp. 311-313, 2005, DOI:10.1109/LED.2005.846578.

[8] V. Janardhanam, H. K. Lee, K. H. Shim, H. B. Hong, S. H. Lee, K. S. Ahn, and C. J. Choi, "Temperature dependency and carrier transport mechanisms of Ti/p-type InP Schottky rectifiers," J. Alloys Compd., vol. 504, no. 1, pp. 146-150, 2010, DOI:10.1016/j.jallcom.2010.05.074.

[9] H. Zhang, E. J. Miller, and E. T. Yu, "Analysis of leakage current mechanisms in Schottky contacts to $\mathrm{GaN}$ and $\mathrm{Al}[\mathrm{sub} 0.25] \mathrm{Ga}[\mathrm{sub}$ $0.75]$ NGaN grown by molecular-beam epitaxy," J. Appl. Phys., vol. 99, no. 2006, p. 023703, 2006, DOI:10.1063/1.2159547.

[10] L. Aguilera, M. Porti, M. Nafria, and X. Aymerich, "Charge trapping and degradation of $\mathrm{HfO} 2 / \mathrm{SiO} 2 \mathrm{MOS}$ gate stacks observed with enhanced CAFM," IEEE Electron Device Lett., vol. 27, no. 3, pp. 157-159, 2006, DOI:10.1109/LED.2006.869799.

[11] V. Yanev, M. Rommel, M. Lemberger, S. Petersen, B. Amon, T. Erlbacher, A. J. Bauer, H. Ryssel, A. Paskaleva, W. Weinreich, C. Fachmann, J. Heitmann, and U. Schroeder, "Tunneling atomic-force microscopy as a highly sensitive mapping tool for the characterization of film morphology in thin high-k dielectrics," Appl. Phys. Lett., vol. 92, no. 2007, pp. 7-10, 2008 , DOI: $10.1063 / 1.2953068$.

[12] U. Celano, Y. Yin Chen, D. J. Wouters, G. Groeseneken, M Jurczak, and W. Vandervorst, "Filament observation in metal-oxide resistive switching devices," Appl. Phys. Lett., vol. 102, pp. 2013 2016, 2013, DOI:10.1063/1.4798525.

[13] M. Porti, M. Nafria, and X. Aymerich, "Current limited stresses of $\mathrm{SiO} / \mathrm{sub} 2 /$ gate oxides with conductive atomic force microscope," IEEE Trans. Electron Devices, vol. 50, no. 4, pp. 933-940, 2003, DOI:10.1109/TED.2003.812082.

[14] K. Shubhakar, K. L. Pey, N. Raghavan, S. S. Kushvaha, M. Bosman, Z. Wang, and S. J. O'Shea, "Study of preferential localized degradation and breakdown of $\mathrm{HfO} 2 / \mathrm{SiOx}$ dielectric stacks at grain boundary sites of polycrystalline $\mathrm{HfO} 2$ dielectrics," Microelectron. Eng., vol. 109, pp. 364-369, 2013, DOI:10.1016/j.mee.2013.03.021.

[15] Y. L. Wu, J. J. Lin, B. T. Chen, and C. Y. Huang, "Positiondependent nanoscale breakdown characteristics of thin silicon dioxide film subjected to mechanical strain," IEEE Trans. Device Mater. Reliab., vol. 12, no. 1, pp. 158-165, 2012 DOI:10.1109/TDMR.2011.2179804.

[16] M. Porti, M. Nafría, M. C. Blüm, X. Aymerich, and S. Sadewasser, "Atomic force microscope topographical artifacts after the dielectric breakdown of ultrathin SiO2 films," Surf. Sci., vol. 532-535, pp 727-731, 2003, DOI:10.1016/S0039-6028(03)00150-X.
Paccagnella, "Using AFM related techniques for the nanoscale electrical characterization of irradiated ultrathin gate oxides," IEEE Trans. Nucl. Sci., vol. 54, no. 6, pp. 1891-1897, 2007, DOI:10.1109/TNS.2007.909483.

[18] J. C. Moore, J. E. Ortiz, J. Xie, H. Morkoç, and a a Baski, "Study of leakage defects on $\mathrm{GaN}$ films by conductive atomic force microscopy," J. Phys. Conf. Ser., vol. 61, pp. 90-94, 2007, DOI:10.1088/1742-6596/61/1/019.

[19] T. Yokoyama, Y. Kamimura, K. Edagawa, and I. Yonenaga, "Local current conduction due to edge dislocations in deformed $\mathrm{GaN}$ studied by scanning spreading resistance microscopy," Eur. Phys. J. Appl. Phys., vol. 61, p. 10102, 2013, DOI:10.1051/epjap/2012120318.

[20] J. Spradlin, S. Doğan, J. Xie, R. Molnar, a. a. Baski, and H. Morkoç, "Investigation of forward and reverse current conduction in GaN films by conductive atomic force microscopy," Appl. Phys. Lett., vol. 84, no. 2004, pp. 4150-4152, 2004, DOI:10.1063/1.1751609.

[21] B. S. Simpkins, H. Zhang, and E. T. Yu, "Defects in nitride semiconductors: From nanoscale imaging to macroscopic device behavior," Mater. Sci. Semicond. Process., vol. 9, pp. 308-314, 2006, DOI:10.1016/j.mssp.2006.01.025.

[22] V. Iglesias, Q. Wu, M. Porti, M. Nafría, G. Bersuker, and a. Cordes, "Monitoring defects in III-V materials: A nanoscale CAFM study," Microelectron. Eng., vol. 147, pp. 31-36, 2015, DOI:10.1016/j.mee.2015.04.058

[23] V. Iglesias, M. Porti, C. Couso, Q. Wu, S. Claramunt, M. Nafria, E. Miranda, N. Domingo, G. Bersuker, and A. Cordes, "Threading dislocations in III-V semiconductors: Analysis of electrical conduction," in 2015 IEEE International Reliability Physics Symposium, 2015, pages. CD.4.1-CD.4.6. DOI: 10.1109/IRPS.2015.7112788

[24] W. Kruppa and J. B. Boos, "Examination of the Kink Effect in InAlAs / InGaAs / InP HEMTs Using Sinusoidal and Transient Excitation," Trans. electron devices, vol. 42, no. 10, pp. 1717-1723, 1995, DOI:10.1109/16.464427. 